\title{
Participación ciudadana en la radio comunitaria en la región central de Ecuador
}

\author{
Citizen participation in community radio \\ in the central region of Ecuador
}

\author{
Alex H. Mullo López \\ Universidad Técnica de Cotopaxi \\ alex.mullo@utc.edu.ec \\ http://orcid.org/0000-0001-9876-9210 \\ Juan P. Toro Bravo \\ Universidad Técnica de Cotopaxi \\ juan.toro@utc.edu.ec \\ https://orcid.org/0000-0001-6647-0337 \\ Lorena C. Álvarez Garzón \\ Universidad Técnica de Cotopaxi \\ lorena.alvarez@utc.edu.ec \\ http://orcid.org/0000-0002-4055-2729
}

\begin{abstract}
Resumen
El artículo analiza la labor que cumplen las radios comunitarias de la región central de Ecuador a fin de promover el progreso social, mediante el fomento de procesos participativos que garanticen el empoderamiento de la comunidad en la administración y creación de contenidos radiofónicos. De esta manera se utiliza metodologías cualitativas a través de entrevistas aplicadas a 12 directores y 36 locutores, con el fin de identificar cuál es el modelo de gestión de las radios comunitarias, cómo se vincula al personal que labora en dichas emisoras, las tipologías de financiamiento con la cual se solventan, las diversas estrategias de participación en la programación radial y las acciones que ha emprendido el Gobierno de Ecuador en favor de las radios estudiadas. Finalmente, se determina que sumado al conocido problema económico se evidencia que no existen estrategias de comunicación horizontal que faciliten el empoderamiento de los ciudadanos alrededor del medio de comunicación. La Ley Orgánica de Comunicación no diversifica a las radios comunitarias para que en función de sus diferencias se impulse una intervención equitativa en el espectro radioeléctrico. Así mismo no impulsa el empoderamiento de la gente como voluntarios. El tema de capacitación es una necesidad que puede ser satisfecha desde la academia.
\end{abstract}

\section{Palabras clave}

Radio comunitaria, gestión, organización, Ley de comunicación, participación ciudadana.

Forma sugerida de citar: Mullo López, A., Toro Bravo, J., y Álvarez Garzón, L. (2019). Participación ciudadana en la radio comunitaria en la región central de Ecuador. Universitas, 31, pp. 175-196. 


\begin{abstract}
The article analyzes the work done by community radio stations in the central region of Ecuador in order to promote social progress through the promotion of participatory processes that guarantee the empowerment of the community in the administration and creation of radio content. In this way, qualitative methodologies are used through interviews applied to 12 directors and 36 broadcasters in order to identify the management model of community radio stations, how the staff working in these stations are linked, the types of financing with which they are financed, the various strategies for participation in radio programming and the actions undertaken by the Government of Ecuador in favour of the radio stations studied. Finally, it is determined that added to the well-known economic problem, it is evident that there are no horizontal communication strategies that facilitate the empowerment of citizens around the media. The Organic Law on Communication does not diversify community radio stations so that, depending on their differences, an equitable intervention in the radio spectrum is promoted. Likewise, it does not promote the empowerment of people as volunteers. The issue of training is a need that can be satisfied from the academy.
\end{abstract}

\title{
Keywords
}

Community radio, management, organization, Law of communication, citizen participation.

\section{Introducción}

Los medios comunitarios, alternativos o ciudadanos surgen de la necesidad de democratizar la palabra en busca de una sociedad más justa, equitativa y solidaria, por lo tanto, son "una herramienta crítica para el control social de los poderes mediáticos tradicionales y para el empoderamiento ciudadano y la participación activa en la esfera pública" (Cerbino \& Belotti, 2016, p. 50). Un manejo del discurso contrario al poder dominante, que faculta "la emancipación de los estratos más bajos de la población frente a la dominación de los estratos superiores" (Beltrán \& Reyes, 1993, p. 19), por medio de una comunicación participativa e incluyente que posibilita el coloquio de saberes para el bienestar común. Visto de esta forma un medio comunitario "no puede existir si no es en función de la dinámica social en la que se desarrolla" (Gumucio, 2005, p. 8). De hecho, el medio se involucra en el contexto social para transmitir en antena una realidad local, "valorizando lo propio, el lugar, 
la identidad, la cultura como herramienta política y de cambio, fomentando la educación colaborativa y de base, las lenguas propias, las formas de organización originarias y, a su vez, abriéndose a un mundo cada vez más conectado" (Ramos, 2018, p. 34). En resumidas cuentas "los medios comunitarios son respuestas puntuales a necesidades sociales" (García \& Ávila, 2016, p. 278). Y dentro de las diversas tipologías de medios alternativos, sobresale la radio, como un espacio ideal para la democratización de la comunicación, mediante la participación oral de los actores sociales. Al mismo tiempo, se destaca la factibilidad económica que tiene toda la población para acceder a dispositivos receptores de señales radiofónicas.

Las radios comunitarias se crean para estimular la participación de una amplia muestra representativa de los diferentes niveles socio-económicos, organizaciones y grupos minoritarios que existen dentro de la comunidad. La misión de estas radios es facilitar la libre circulación de información estimulando la libertad de expresión y el diálogo dentro de la comunidad. (Unesco, 1999, p. 152)

De acuerdo con la Asociación Mundial de Radios Comunitarias (Amarc, 2010), las radios y televisoras comunitarias son:

Actores privados que tienen una finalidad social y se caracterizan por ser gestionadas por organizaciones sociales de diverso tipo sin fines de lucro. Su característica fundamental es la participación de la comunidad tanto en la propiedad del medio como en la programación, administración, operación, financiamiento y evaluación. (p. 51)

Desde la perspectiva del cubano José Ignacio López, una emisora comunitaria:

Promueve la participación de los ciudadanos y defiende sus intereses; cuando responde a los gustos de la mayoría y hace del buen humor y la esperanza su primera propuesta; cuando informa verazmente; cuando ayuda a resolver los mil y un problemas de la vida cotidiana; cuando en sus programas se debaten todas las ideas y se respetan todas las opiniones; cuando se estimula la diversidad cultural y no la homogeneización mercantil. (1995, p. 54)

Dicho de otro modo, una radio ciudadana, deberá siempre estar abierta a su audiencia y no podrá prescindir del vínculo con su comunidad, ya que "apunta siempre hacia la democratización de la comunicación y hace de la 
participación ciudadana un elemento primordial" (Pincheira, 2013, p. 184). Favoreciendo siempre una comunicación horizontal que "debe articularse en el sentido que el radioescucha reclama su gratificación, quiere ser productor y destinatario de la información" (Abreu et al., 2010, p. 22). Permitiendo así, promover el bienestar y desarrollo de la colectividad.

Volviendo la mirada hacia el pasado, en América Latina las radios comunitarias aparecen en la "primera mitad del siglo XX, al amparo de movimientos sociales y políticos, ligados a sindicatos y movimientos católicos progresistas" (Ramírez, 2014,p. 119) que dieron paso a un gran proceso de construcción mediática educativa que nació en Colombia, a través de las ondas Hertzianas de Radio Sutatenza. Proyecto que "por más de 50 años, entre 1948 y 1990, benefició a cerca de ocho millones de analfabetas, manejando sus cinco ejes esenciales (salud, alfabeto, números, trabajo espiritualidad)" (Arnedo et al., 2014, p. 242). De igual manera aparecen en los años 50, las emisoras bolivianas cercanas al movimiento sindical minero. Propuesta radial que nace por la decisión de un "grupo de sacerdotes comprometidos con el sector. La misma consistió en lanzar una radio - que se convertiría en varias - en la que se escucharan las voces de los mineros y sus familias" (Villamayor, 2014, p. 91).

Posteriormente en los años 60 surgen las Escuelas Radiofónicas Populares del Ecuador (ERPE), las cuales generaron "la creación de cerca de medio millar de emisoras, dedicadas a la educación popular, en su mayoría patrocinadas por la Iglesia Católica" (Beltrán, 1996, p. 9). Finalmente, ERPE "en la década del 80 abandonó su origen alfabetizador y se vinculó a la idea de cambio de las estructuras de la sociedad y la búsqueda de una sociedad justa, con solidaridad y equidad" (Villamayor, 2014, p. 91). Evidentemente la radio comunitaria se expone como una propuesta emancipadora de los pueblos, que apertura canales comunicacionales para el diálogo de saberes "desde una perspectiva política que involucra a la comunidad y no a los intereses del mercado o los poderes hegemónicos" (Lizondo, 2018, p. 64).

De esta manera:

Los actores pueden apropiarse legítimamente de una información que ellos mismos construyen con las demandas y con las experiencias que parten desde su realidad social, y que no es impuesta por medios que construyen la noticia desde otros ámbitos. (Navarro, 2017, p. 11- 12)

Una comunicación que se erige en lo local, en lo cotidiano, por ello "se considera como un medio cercano y de gran carga emocional" (Martínez \& 
Ortega, 2018, p. 87). Sobre las ideas expuestas, este trabajo pretende analizar la labor que cumplen las radios comunitarias de la región central de Ecuador, para promover el desarrollo social, mediante la apertura de procesos participativos que permitan a los actores sociales comprometerse en la administración (modelo de gestión), operación (personal que labora), financiamiento (sostenibilidad económica) y programación del medio comunitario (estrategias de participación en la creación de contenidos). Finalmente, se indagará el apoyo estatal que han recibido las radios comunitarias con el objetivo de determinar las acciones emprendidas por el Gobierno ecuatoriano en favor de las emisoras.

\section{Participación ciudadana en la radio comunitaria}

La participación social es una clave fundamental para la construcción de una radio comunitaria, esto se evidencia desde las primeras iniciativas latinoamericanas. Tal es el caso de la radio minera, la cual promovía la participación mediante "su estrategia de 'micrófono abierto', ponían a los trabajadores y a sus familias a hablar libremente de todos los temas de su interés, a hacer reclamos a las autoridades e inclusive a criticar la conducción sindical" (Beltrán, 1996, p. 9). De ahí que la emisora comunitaria es un medio que se involucra con su comunidad, para propender al diálogo que fomente una sociedad crítica contra el poder de turno, a fin de que no solo sea "un canal de transmisión a la gente, sino también un medio de recepción de ellos" (Milan, 2006 p. 272). De esta manera la participación ciudadana en la radio comunitaria "debe ser vista en el análisis del espacio público como capacidad y posibilidad de las personas y grupos sociales de influir en la transformación de las condiciones que afectan la propia vida" (Martínez, Yaguana \& Rencoret, 2018, p. 19).

Ahora bien, existen diversas tipologías de participación en la radio comunitaria, sin embargo, todas están enfocadas a la democratización de los medios. La participación desde la gestión impulsa a la comunidad a involucrarse en la toma de decisiones en la administración de la emisora. Con esta finalidad se ejecuta una comunicación horizontal para la contratación del personal, el nombramiento del administrador o director, el mantenimiento de equipos e instalaciones, entre otras. Otra tarea prioritaria en este nivel de participación es la contribución de la comunidad para la sostenibilidad económica del medio. 
Por consiguiente, una radio comunitaria debe "generar espacios para la participación de los ciudadanos desde la gestión, cogestión y autogestión, como los niveles más elevados de compromisos con la pluralidad y libertad de expresión para la consecución de los mejores intereses de las comunidades (Mora, 2011, p .140). Mientras que, la participación desde el ámbito de la gestión de contenidos implica la elaboración del proyecto comunicacional y la toma de decisiones en cuanto a las temáticas que serán transmitidas en la parrilla de programación. Para ello el medio de comunicación debe generar estrategias que permitan a la localidad vincularse en la producción y emisión de programas radiofónicos que contribuyan al desarrollo social y la promoción intercultural.

Por último, la participación en la recepción y retroalimentación de los programas radiales, "mediante la expresión de opiniones, la generación de cuestionamientos que amplían la comprensión de los hechos" (De la Noval, 2018, p. 36), son elementos fundamentales para la subsistencia del medio. La sintonía de los habitantes de la comunidad permite fortalecer la razón de ser del medio de comunicación. Una emisora que nace de "una necesidad específica de la comunidad, o como paso lógico de un proceso de desarrollo comunitario, será una emisora en la que la comunidad participe" (Álvarez, 2008 , p. 68). La participación por tanto es un compromiso bidireccional, por un lado, el medio tiene la responsabilidad de apoyar a su comunidad para popularizar la palabra, a su vez la comunidad mantiene el deber de apoyar al medio en la gestión administrativa, la emisión y recepción del producto comunicacional. En esta perspectiva:

La radio debe convertirse en un espacio de participación de las comunidades y solo la vinculación efectiva con las mismas garantizará el soporte necesario para que dichas comunidades defiendan su radio como herramienta comunicacional para el desarrollo de sus capacidades y para el mejoramiento de su propia vida en comunidad. (Romero \& Artigas, 2013, p. 77)

La ventaja del medio comunitario radica no solo en su cercanía geográfica con la colectividad, sino en una cercanía comunicacional que permite a los emisores radiofónicos escuchar y ser escuchados por la sociedad. De allí pues, que el éxito se logra:

En la medida que los pueblos y organizaciones sean los protagonistas en la producción y circulación de mensajes, que rompan los cánones establecidos 
por los medios de comunicación empresariales, que reproducen en sus agendas la pirámide del dominio, donde los políticos, artistas y deportistas son importantes. (Galán, 2015, p. 30)

Dicho de otro modo, los medios comunitarios no se sirven del pueblo, por el contrario, sirven al pueblo dando "cabida a nuevas problemáticas y perfiles de participantes, siendo los grupos y colectivos que más sufren la exclusión, o con mayores dificultades en el ejercicio de sus derechos, los que muestran mayor interés en participar" (García, 2017, p. 40). Por último, es conveniente anotar que, las radios comunitarias son las llamadas a dar el primer paso para crear vínculos que permitan la cooperación y el compromiso de la población para trabajar por y para el medio de comunicación, recuperando "el protagonismo de la ciudadanía en el proceso comunicativo, incluyendo la elaboración de contenidos y la gestión del propio medio de comunicación" (Martínez, 2018, p. 271).

De esta manera las comunidades se empoderarán de la radio local comunitaria y fortalecerán su desarrollo social mediante procesos comunicacionales. De hecho:

Cuando las comunidades se apropian del medio de comunicación, descubren lo útil que pueden llegar a ser para tejer de manera más sólida sus procesos y lograr visibilizar su realidad y su contexto contados en sus propios términos. (Peña, 2012, p. 200)

\section{La radio comunitaria y su marco legal en Ecuador}

Las primeras radios comunitarias surgen sin un marco regulatorio del Estado y gracias al apoyo de la iglesia católica. En 1950 de la mano del Reverendo Manuel Moncayo, Administrador Apostólico del Vicariato de Zamora, se funda radio la Voz de Zamora, en la provincia de Zamora Chinchipe, la cual fue creada para el servicio de la fe, y el incremento de la justicia y la difusión de la cultura (http://lavozdezamora.com.ec). De modo similar en 1962 y por iniciativa de Monseñor Leónidas Proaño, surge en Riobamba, las Escuelas Radiofónicas Populares del Ecuador (ERPE), proyecto radial que se enmarcaba en la teología de la liberación y que buscaba la justicia social para los pueblos indígenas de la región. Mediante el impulso de: 
Programas de alfabetización, evangelización y educación de los pueblos indígenas y campesinos no considerados por el Estado. El programa promovió la movilización de los campesinos ecuatorianos hacia la defensa de su identidad y el combate a la desigualdad y pobreza. (Sanmartín et al., 2017, p. 101)

Subsiguientemente, en la provincia de Morona Santiago, ubicada en la región amazónica, surge radio La Voz de Arutam. “Esta empezó en 1964 con la misión salesiana, y en los años setenta desarrolló el Sistema de Educación Radiofónico Bilingüe Intercultural Shuar (Serbish) para el nivel primario" (Acosta et al., 2017, p. 7). Años más tarde en 1972, la Fundación Adelanto Comunitario Ecuatoriano emprende la creación de radio Interoceánica, "institución de comunicación y servicio a la comunidad marcando un modelo de vida en la fe y compromiso del pueblo" (Cerbino, 2018, p. 121). Ya a partir de 1975 se instaura la ley de radiodifusión y televisión que reconocía solo dos tipos de medios, las estaciones públicas y las estaciones comerciales privadas. Por ende, a partir de este marco regulatorio las radios comunitarias que estaban en funcionamiento y las propuestas nuevas surgirían como emisoras privadas.

Ulteriormente, aunque con ciertas limitantes, en el año de 1995, por fin se implementan las concesiones de radiodifusión comunales, para las organizaciones legalmente constituidas, de acuerdo con la Ley de Organización y Régimen de las Comunas. Para el año 2013 se crearía la Ley Orgánica de Comunicación (LOC), que reconoce tres tipologías de medios de comunicación social, siendo estos públicos, privados y comunitarios. De acuerdo con la sección III sobre los medios de comunicación comunitarios, la LOC en su artículo 85 define a los medios comunitarios como "aquellos cuya propiedad, administración y dirección corresponden a colectivos u organizaciones sociales sin fines de lucro, a comunas, comunidades, pueblos y nacionalidades. Los medios de comunicación comunitarios no tienen fines de lucro y su rentabilidad es social" (p.15). Dentro de esta perspectiva se plantea entonces el problema. ¿De qué manera podrían solventar los gastos que conlleva el mantenimiento de una estación radial y su misión social, si no tienen fines de lucro?

Por otra parte, el artículo 110 de la LOC enuncia que los medios comunitarios deben concursar con los privados para poder adquirir una frecuencia del espectro radioeléctrico cumpliendo con requisitos como, el proyecto comunicacional, estudio técnico y de gestión y sostenibilidad. Lo cual de- 
mandaría de una fuerte inversión económica para una comunidad, pueblo o nacionalidad. A esto se suma la complejidad que tienen los voluntarios de las propias comunidades para colaborar en las actividades periodísticas del medio comunitario, ya que según el artículo 42 de la LOC, para laborar en un medio de comunicación social se debe ser profesional de periodismo o comunicación a excepción de los colaboradores que realicen programas en lenguas nativas.

Las radios comunitarias son una herramienta para impulsar la participación de los pueblos y comunidades:

Porque a través de ella tendrán la posibilidad de incidir en las decisiones públicas y privadas en lo comunitario (familia y escuela) y local, promoviendo y exigiendo el cumplimiento de sus derechos, mediante la organización, movilización, comunicación y formación. (Barragán, \& Garzón, 2017, p. 290)

Sin duda:

Se nota una falta de comunicación y trabajo integrado con los sectores comunitarios en la construcción de las leyes, lo cual no permite que el Estado pueda comprender las implicaciones, necesidades y características propias y particulares de las radios comunitarias. (Galarza \& Culqui, 2017, p. 1405)

Y es en estas "diferencias donde se debe trabajar para alcanzar la democratización de los medios para dar voz a los que tradicionalmente han estado excluidos del debate público” (Barragán \& Garzón, 2016, p. 212).

\section{Material y métodos}

El estudio se realiza en las radios comunitarias de la región central del Ecuador, que comprenden las provincias de Cotopaxi, Tungurahua, Chimborazo y Pastaza. La metodología utilizada para el proceso de recolección de datos es de carácter cualitativo, mediante la técnica de la entrevista semiestructurada a 12 directores y 36 locutores de dichos medios. En función de las siguientes variables de estudio: a) Modelo de gestión de las radios estudiadas; b) Personal que forma parte de las radios comunitarias; c) Sostenibilidad económica en las radios analizadas; d) Estrategias de participación en la programación radial; e) Apoyo estatal para el fortalecimiento de la radio comunitaria. 
Se analiza el modelo de gestión de las radios estudiadas, con el fin de identificar a quien o a quienes pertenece el medio radial. Las personas que intervienen en la administración de la radio comunitaria, y el objetivo de la programación como tal. Seguidamente el personal que forma parte de las emisoras, con el propósito de establecer cómo se contrata y vincula a las personas que laboran en la radiodifusora. Así mismo el número de personas trabajan voluntariamente en el medio de comunicación. En tanto que, la sostenibilidad económica de las radios comunitarias es examinada con la intención de conocer las diversas tipologías de financiamiento con la cual se solventa el medio radial.

Posteriormente, los diversos mecanismos que utilizan las emisoras comunitarias para propender a la participación de la comunidad en el proyecto comunicacional. Finalmente, el apoyo estatal para el fortalecimiento de la radio Comunitaria se lo investiga con el objetivo de determinar las acciones que ha emprendido el Gobierno de Ecuador en favor de las emisoras estudiadas. En cuanto al uso de la metodología propuesta, está tiene ciertas limitaciones, como la subjetividad en los resultados cualitativos, ya que se toma como referencia a la radio comunitaria de la región central del Ecuador desde el criterio de sus directores y locutores.

\section{Análisis y resultados}

\section{Modelo de gestión de las radios estudiadas}

Para entender el modelo de gestión de las radios comunitarias se realizan entrevistas a 12 directores en función de las siguientes categorías:

- A quién o a quiénes pertenece el medio radial.

- Las personas que intervienen en la administración del medio y los objetivos de la programación de las emisoras.

\section{Identificar a quién o a quiénes pertenece el medio radial}

El estudio determina que siete emisoras pertenecen a organizaciones religiosas, dos a una fundación privada sin fines de lucro, y cinco a pueblos 
y nacionalidades indígenas. No obstante, de las radios comunitarias que se investigaron, solo nueve están en funcionamiento. Como ilustra la tabla 1.

Las personas que intervienen en la administración de la radio comunitaria

Al analizar las dinámicas respecto a la administración, los hallazgos determinan que las emisoras bajo la tutela de grupos religiosos tienen administradores y coordinadores nombrados por las autoridades de dichas comunidades, mientras que, en las emisoras que pertenecen a grupos indígenas y a fundaciones privadas, los administradores son elegidos por una Asamblea general.

\section{Los objetivos de la programación radial comunitaria}

Referente a los objetivos que rigen la programación de las radios comunitarias, de acuerdo con el estudio se determina que estos están armonizados a la razón social de cada una, es decir se enfocan en evangelizar, satisfacer las necesidades sociales de la población marginada, y fortalecer la comunicación en el aspecto político, cultural y social dentro de la comunidad. Para las emisoras la sostenibilidad es un tema sensible, de los entrevistados se desprende que quienes cerraron debieron hacerlo por la falta de recursos económicos para enfrentar gastos como pago de energía y cánones de arriendo.

\section{Tabla 1}

\section{Radios comunitarias de la región central del Ecuador registradas en el CORDICOM}

\begin{tabular}{|l|l|l|l|l|}
\hline \multicolumn{1}{|c|}{ Razón social } & \multicolumn{1}{|c|}{$\begin{array}{c}\text { Nombre del } \\
\text { medio }\end{array}$} & $\begin{array}{c}\text { Frecuencia/ } \\
\text { canal }\end{array}$ & \multicolumn{1}{|c|}{ Provincia } & $\begin{array}{c}\text { En funciona- } \\
\text { miento }\end{array}$ \\
\hline $\begin{array}{l}\text { Federación de iglesias } \\
\text { indígenas evangélicas } \\
\text { de Cotopaxi }\end{array}$ & Radio Runatacuyac & $1160 \mathrm{AM}$ & Cotopaxi & Sí \\
\hline $\begin{array}{l}\text { Fundación ecuatoriana } \\
\text { Juan Pablo II }\end{array}$ & $\begin{array}{l}\text { Radio católica } \\
\text { Riobamba }\end{array}$ & $105.7 \mathrm{FM}$ & Chimborazo & Sí \\
\hline $\begin{array}{l}\text { Asociación de } \\
\text { comunidades indígenas } \\
\text { de Arajuno }\end{array}$ & $\begin{array}{l}\text { Radio Jatari } \\
\text { kichwa 92.3 FM }\end{array}$ & $92.3 \mathrm{FM}$ & Pastaza & Sí \\
\hline $\begin{array}{l}\text { Nación Sapara } \\
\text { del Ecuador }\end{array}$ & $\begin{array}{l}\text { Radio Sapara } 92.7 \\
\text { FM }\end{array}$ & $92.7 \mathrm{FM}$ & Pastaza & No \\
\hline
\end{tabular}




\begin{tabular}{|c|c|c|c|c|}
\hline $\begin{array}{l}\text { Nacionalidad Andwa de } \\
\text { Pastaza Ecuador }\end{array}$ & $\begin{array}{l}\text { Radio Andwa la } \\
\text { voz de la frontera }\end{array}$ & $95.9 \mathrm{FM}$ & Pastaza & No \\
\hline $\begin{array}{l}\text { Nacionalidad Waorani del } \\
\text { Ecuador }\end{array}$ & $\begin{array}{l}\text { Wao apeninka } 91.1 \\
\text { FM }\end{array}$ & $91.1 \mathrm{FM}$ & Pastaza & No \\
\hline $\begin{array}{l}\text { Nacionalidad Shiwiar del } \\
\text { Ecuador }\end{array}$ & $\begin{array}{l}\text { Radio Tarimiat } \\
93.5 \mathrm{fm}\end{array}$ & $93.5 \mathrm{FM}$ & Pastaza & No \\
\hline $\begin{array}{l}\text { Fundación escuelas } \\
\text { radiofónicas populares }\end{array}$ & $\begin{array}{l}\text { Fundación escuelas } \\
\text { radiofónicas } \\
\text { populares }\end{array}$ & $710 \mathrm{AM}$ & Chimborazo & No \\
\hline $\begin{array}{l}\text { Fundación escuelas } \\
\text { radiofónicas populares }\end{array}$ & $\begin{array}{l}\text { Fundación escuelas } \\
\text { radiofónicas } \\
\text { populares }\end{array}$ & $91.7 \mathrm{FM}$ & Chimborazo & Sí \\
\hline Diócesis de Latacunga & $\begin{array}{l}\text { Radio Latacunga } \\
\text { FM }\end{array}$ & 102.1 FM & Cotopaxi & Sí \\
\hline $\begin{array}{l}\text { Vicariato apostólico de } \\
\text { Puyo misión dominicana }\end{array}$ & Radio Puyo & $89.1 \mathrm{FM}$ & Pastaza & Sí \\
\hline $\begin{array}{l}\text { Asociación indígena de } \\
\text { la iglesia de cristo en } \\
\text { Chimborazo }\end{array}$ & El Prado & $980 \mathrm{AM}$ & Chimborazo & Sí \\
\hline $\begin{array}{l}\text { Confederación de } \\
\text { pueblos organizaciones } \\
\text { comunidades e iglesias } \\
\text { indígenas evangélicas de } \\
\text { Chimborazo }\end{array}$ & La voz de AIIECH & $950 \mathrm{AM}$ & Chimborazo & Sí \\
\hline $\begin{array}{l}\text { Confederación de } \\
\text { pueblos organizaciones } \\
\text { comunidades e iglesias } \\
\text { indígenas evangélicas de } \\
\text { Chimborazo }\end{array}$ & La voz de AIIECH & $101.7 \mathrm{FM}$ & Chimborazo & Sí \\
\hline
\end{tabular}

Fuente: Elaboración propia

\section{Personal de las radios comunitarias}

De acuerdo con los 12 directores y los 36 locutores de las emisoras comunitarias en funcionamiento, el personal que trabaja y colabora en la radio se vincula mediante contratos y acuerdos verbales o escritos. Los contra- 
tos son debidamente legalizados en el Ministerio de Relaciones Laborales. Mientras que, los acuerdos escritos o verbales se dan con colaboradores o voluntarios que apoyan en todo tipo de actividades comunicacionales, desempeñándose como locutores y reporteros comunitarios. Cabe destacar que los voluntarios son personas que pertenecen a la comunidad, fundación u organización propietaria de la emisora. De esta manera la radio se transforma en un espacio que no les genera réditos económicos, pero si satisfacciones de apoyo a la comunidad. De ahí se entiende el número elevado de voluntarios en varias emisoras. Así lo ilustra la tabla 2.

Tabla 2

Personal que forma parte de las radios comunitarias

\begin{tabular}{|c|c|c|c|}
\hline \multirow{2}{*}{ Radio } & \multirow{2}{*}{$\begin{array}{l}\text { Número de } \\
\text { colaboradores }\end{array}$} & $\begin{array}{l}\text { Número de } \\
\text { voluntarios: }\end{array}$ & $\begin{array}{l}\text { Número de personas } \\
\text { contratadas: }\end{array}$ \\
\hline & & $\begin{array}{l}\text { Mediante acuerdos } \\
\text { escritos o verbales }\end{array}$ & Mediante remuneración \\
\hline $\begin{array}{l}\text { Radio Runatacuyac } \\
1160 \text { AM }\end{array}$ & 5 & 3 & 2 \\
\hline $\begin{array}{l}\text { Radio católica Riobamba } \\
\text { 105.7 FM }\end{array}$ & 14 & 12 & 2 \\
\hline $\begin{array}{l}\text { Radio Jatari kichwa } \\
92.3 \text { FM }\end{array}$ & 10 & 10 & \\
\hline $\begin{array}{l}\text { Fundación escuelas } \\
\text { radiofónicas populares } \\
91.7 \text { FM }\end{array}$ & 59 & 50 & 9 \\
\hline $\begin{array}{l}\text { Radio Latacunga } \\
\text { 102.1 FM }\end{array}$ & 11 & & 11 \\
\hline Radio Puyo 89.1 FM & 17 & 13 & 4 \\
\hline El Prado 980 AM & 10 & 10 & \\
\hline $\begin{array}{l}\text { La voz de AIIECH } \\
950 \text { AM y } 101.7 \text { FM }\end{array}$ & 20 & 17 & 3 \\
\hline Total & 146 & 115 & 31 \\
\hline
\end{tabular}

Fuente: Elaboración propia 


\section{Sostenibilidad económica en las radios comunitarias}

La sostenibilidad económica es un tema sensible y trascendental para las emisoras, según los 12 directores de los medios estudiados, mantener operativa a una radio comunitaria requieren una inversión y gasto mensual aproximado entre 5000 a 10000 dólares americanos.

El financiamiento para pagos de servicios básicos y al personal que colabora en el medio, se sustenta por la publicidad de instituciones públicas y privadas entre otros. No obstante, algunas emisoras restringen los contenidos publicitarios que contengan mensajes en contra de sus creencias religiosas o principios éticos y morales. En consecuencia, buscan generar recursos económicos mediante emprendimientos socio productivos o servicios de transmisión de eventos, así como proyectos internacionales.

Por otra parte, las donaciones son realizadas por personas de la organización, fundación o comunidad a la pertenece la emisora. Las donaciones de otros países, que en determinado tiempo fueron un gran aporte de las emisoras, han sido limitadas por los trámites burocráticos impuestos por el estado ecuatoriano. A continuación, en la tabla 3 se expone las principales fuentes de financiamiento de las radios comunitarias de la región central de Ecuador.

Tabla 3

Sostenibilidad económica en las radios comunitarias

\begin{tabular}{|c|c|c|c|}
\hline \multirow{2}{*}{ Radio } & \multicolumn{3}{|c|}{ Principales fuentes de financiamiento } \\
\hline & Publicidad & Donaciones & Otros \\
\hline Radio Runatacuyac1160 AM & & $\mathrm{X}$ & \\
\hline Radio católica Riobamba 105.7 FM & $\mathrm{X}$ & & \\
\hline Radio Jatari kichwa 92.3 FM & $\mathrm{X}$ & & \\
\hline $\begin{array}{l}\text { Fundación escuelas radiofónicas } \\
\text { populares } 91.7 \mathrm{FM}\end{array}$ & $\mathrm{X}$ & & $\begin{array}{l}\text { Ingresos de una granja } \\
\text { agrícola y tienda orgánica }\end{array}$ \\
\hline Radio Latacunga 102.1 FM & $\mathrm{X}$ & $\mathrm{X}$ & $\begin{array}{l}\text { Transmisiones en vivo } \\
\text { de eventos }\end{array}$ \\
\hline Radio Puyo 89.1 FM & $\mathrm{X}$ & & \\
\hline El Prado 980 AM & & $\mathrm{X}$ & \\
\hline La voz de AIIECH 950 AM y 101.7 FM & & $\mathrm{X}$ & $\begin{array}{l}\text { Transmisiones en vivo } \\
\text { de eventos }\end{array}$ \\
\hline
\end{tabular}

Fuente: Elaboración propia 


\section{Estrategias de participación en la programación radial}

Con referencia a las radios de la provincia de Pastaza. Tanto Radio Puyo y Jatari Kichwa promueven la participación de las personas de la comunidad mediante la transmisión de información local y de la tradición cultural. De esta manera se busca incentivar la retroalimentación de los radioescuchas, para que emitan su criterio en torno a la información difundida, utilizando llamadas telefónicas, mensajes a WhatsApp o redes sociales. Anteriormente se realizaban programas con la comunidad, pero, por falta de recursos económicos y de personal periodístico y voluntario, esta labor fue suspendida. Mientras tanto que, en la provincia de Cotopaxi. Radio Runatacuyac mantiene espacios para jornadas de alabanzas y reflexiones de doctrinas cristianas, con la participación de los feligreses que conforman la Federación de iglesias indígenas evangélicas de Cotopaxi. Por su parte, Radio Latacunga realiza sondeos en las comunidades para establecer los contenidos que requiere la población. Al mismo tiempo, involucra a personas de la misma comunidad para que sean reporteros o corresponsales comunitarios e informen sobre las diversas actividades o necesidades de la ciudadanía. Por último, en la provincia de Chimborazo. Radio ERPE, se involucra con la comunidad para conocer las diversas necesidades informativas y de contenidos educomunicacionales que requiere la población. Para luego diversificarlas en el diseño de la parrilla de programación. Además, priorizan los espacios para los sectores vulnerables. De modo que, personas con discapacidad y estudiantes de unidades educativas mantienen programas al aire.

Con respecto a radio El Prado, las estrategias de participación se enmarcan en la vida micro social de los sectores rurales, es decir, se busca la información local contada por los líderes de las comunidades, para luego generar una retroalimentación de la población, mediante llamadas telefónicas o comunicados escritos. Radio Católica, tiene una política de apertura de micrófonos a quien necesite expresarse. No generan procesos propios de cooperación desde los emisores radiofónicos. Finalmente, Radio La voz de AIIECH crea métodos de participación, incluyendo a locutores que pertenecen a la congregación propietaria de la emisora. De este modo, participan pastores, líderes e integrantes de las diversas iglesias, quienes difunden programas de doctrina religiosa y de contenido educativo. Así lo afirman los directores y locutores de las emisoras estudiadas. 


\section{Apoyo estatal para el fortalecimiento de la radio comunitaria}

Para el análisis del apoyo estatal para el fortalecimiento de la radio comunitaria, se requiere dividir a los medios comunitarios en:

- Radios comunitarias de pueblos y nacionalidades indígenas

- Radios de comunidades religiosas

- Radios comunitarias pertenecientes a fundaciones de interés social.

En los hallazgos se ha verificado que el apoyo estatal a los medios comunitarios de los pueblos y nacionalidades indígenas tiene dos momentos específicos: El primero, el aporte económico del gobierno del expresidente Rafael Correa con el propósito de adquirir equipos tecnológicos para el funcionamiento de las emisoras, y por otro lado la capacitación en el ámbito de la comunicación a los representantes de las comunidades indígenas que colaboraron al inicio del proyecto comunicacional.

Un segundo momento, es el actual, en el que los medios comunitarios no han recibido un apoyo estatal en el ámbito económico, de capacitación administrativa y comunicacional. A esto se suma, la dimisión de los primeros comuneros que fueron capacitados, quienes decidieron abandonar el proyecto por la falta de recursos económicos que les permita costear las necesidades básicas de sus familias. Como resultado de estas dificultades, la mayoría de los medios comunitarios de las nacionalidades indígenas han cerrado o suspendido sus transmisiones. Desde la perspectiva de los directores y locutores de los medios comunitarios de las comunidades religiosas y de fundaciones de interés social. El apoyo estatal ha existido mediante pocos contratos publicitarios con entidades gubernamentales, que han significado un limitado apoyo económico para la sustentabilidad del medio comunitario. En cuanto al aporte para el perfeccionamiento profesional, se indica que no ha existido el apoyo para la capacitación de los colaboradores de los medios radiales.

\section{Discusión y conclusiones}

En la región central de Ecuador, la mayoría de las emisoras pertenecen a organizaciones religiosas y únicamente en la provincia de Pastaza, los pueblos y nacionalidades indígenas han logrado acceder a una frecuencia de 
radio. Dichos medios han enfrentado dificultades económicas y de capacitación lo cual ha dado como resultado que, de los cincos emisoras comunitarias tan solo una siga en funcionamiento. Por ende:

Las comunidades siguen ajenas a la acción estatal y de los medios en tanto no han sido invitadas a esta toma de decisiones, desde donde se puedan incentivar políticas de consolidación de sus formas de existencia, de capacitación formativa y mediática y aquellas legislaciones que dignifiquen su existencia. (Tamarit, Cevallos \& Yépez, 2014, p. 21)

A pesar de que una gran parte de la población indígena del Ecuador se asienta en las provincias de Cotopaxi, Tungurahua y Chimborazo, no existen emisoras que sean propiedad de las nacionalidades indígenas, Al respecto, Sánchez (2013) manifiesta que "en el caso ecuatoriano se tomó el modelo boliviano del 34\% para la comunicación sin fines de lucro, pero sin incidir en la diferenciación entre organizaciones no gubernamentales y nacionalidades o pueblos originarios" (p. 166). La sostenibilidad económica es uno de los principales problemas que afrontan las emisoras comunitarias. De cierta manera, la esencia y objetivos de las radios han restringido el acceso a pautas publicitarias rentables, que podrían atentar a sus creencias o códigos morales.

Por otro lado, se debe analizar que las empresas buscan publicitar en los medios que capten la atención de públicos objetivos con poder adquisitivo. En cambio, los medios comunitarios se deben a las comunidades históricamente excluidas, que tienen como fin el bienestar común. Otro elemento para tomar en cuenta es la vinculación del personal que colabora en los medios comunitarios. Ya que, a diferencia de los medios privados o públicos que contratan personal profesional, los medios comunitarios se apoyan principalmente en voluntarios empíricos.

Asimismo, no existe una participación y compromiso de la comunidad en la radio, específicamente en el ámbito administrativo, financiero y comunicacional. Las personas que intervienen en la administración y manejo financiero de las emisoras son nombradas por autoridades de las organizaciones o comunidades a las que pertenece la emisora. En tanto que, la construcción de los contenidos para la parrilla de programación es decidida mediante encuestas a la población y bajo el criterio de autoridades, en conjunto con los directores del medio. 
De cierta manera en la mayoría de las emisoras, la población se mantiene al margen de la propuesta comunicacional. Realidad que concuerda con la presentada por Viviana Galarza y Belén Amador en la investigación "La radio comunitaria en Imbabura: emisoras, programación y compromiso con la audiencia" en la cual concluyen que "la Radio Comunitaria en la provincia de Imbabura es un medio con potencial, pero se ha olvidado el principal objetivo del medio, la participación de la comunidad" (2018, p. 76). Por tanto, existe una participación vertical, en donde el emisor radial propone cuando y porque medio participará la población. Sin embargo, hay que destacar iniciativas como las de Radio Latacunga y las Escuelas Radiofónicas y Populares del Ecuador, que han permitido involucrar a los propios comuneros para el trabajo comunicacional, desde sus realidades y en su propio idioma. Al mismo tiempo han adjudicado espacios para sectores vulnerables históricamente excluidos de los espacios radiales.

En consecuencia, es necesario resignificar las propuestas comunicacionales de las comunidades y organizaciones sociales:

Desde un ejercicio de regresión que lleve a las Radios Comunitarias al punto de partida, de valorar a una audiencia propositiva, crítica, con puntos de convergencia y desacuerdos, crecida, diversa, con cosmovisiones e identidades particulares, con contradicciones y culturas diferentes, rezándole al Dios de su preferencia, solidario, conflictivo, pasivo, activo, pobre, rico. (Paiz, 2016, p. 104)

En otro orden de ideas, es primordial que en la Ley Orgánica de Comunicación de Ecuador se instaure una diferencia clara entre radios comunitarias religiosas, de organizaciones sin fines de lucro y emisoras comunitarias de comunidades y nacionalidades indígenas. Para que, en función de sus diferencias se fomente una participación equitativa en el espectro radioeléctrico. Igualmente, desde el marco legal se debe promover la colaboración de los propios comuneros como voluntarios, para así fomentar la participación ciudadana y cumplir con la misión social del medio radial. Además, el Estado debe crear políticas tangibles que faculten la sostenibilidad económica de las emisoras. De ahí que esta tipología de medios tiene un fin económico para solventar los fines sociales. De igual manera, los líderes de las comunidades, organizaciones o nacionalidades deben crear estrategias de comunicación horizontal que permitan involucrar a la ciudadanía en el diálogo de saberes para el bien común. Finalmente, el Gobierno ecuatoriano debe apo- 
yarse en las universidades, para que a través de procesos de vinculación se generen capacitaciones técnicas, administrativas, financieras y comunicacionales dirigidas no solamente al personal de los medios de comunicación, sino en sí, a toda la población a la que se adjudique la frecuencia. A fin de que puedan entender las bondades de la comunicación en favor del desarrollo local y se empoderen de sus procesos de sostenibilidad económica, social y comunicacional.

\section{Bibliografía}

Abreu, N., \& Castro, E., \& Plumacher, P., \& Reyes, M. (2010). Situación legal de las radios comunitarias en el municipio Maracaibo del Estado Zulia. Quórum Académico, 7(2), 11-32. Recuperado de: https://goo.gl/u6KTFj

Acosta, A., Calvopiña, V., \& Cano, J. (2017). Medios comunitarios y democratización de la comunicación en Ecuador: aporte para el debate sobre el Concurso Público de Frecuencias, 1-60. Recuperado de: https://goo.gl/zxAZsb

Álvarez Moreno, M. (2008). El desafío de las radios comunitarias. Anagramas Rumbos y Sentidos de la Comunicación, 6(12), 61-77. Recuperado de: https://goo.gl/ePJ5WQ

Amarc (2010). Principios para garantizar la diversidad y el pluralismo en la radiodifusión y los servicios de comunicación audiovisual. Recuperado de: https://goo.gl/BzVbAC

Arnedo, B., Hernández, D., \& Jurado, J. (2014). El laberinto de las radios comunitarias en Bolívar: entre la subsistencia y el aporte social al desarrollo comunitario. Palobra: Palabra que obra, (14), 238-256. Recuperado de: https://goo.gl/jWDr3k

Asamblea Nacional del Ecuador (2013). Ley Orgánica de Comunicación. Quito Ecuador.

Barragán V. \& Garzón, B. (2016). La interculturalidad en las radios comunitarias del Ecuador, las radios de las nacionalidades. Redes.com: Revista de estudios para el desarrollo social de la Comunicación, 4. 194-215. Recuperado de: https://goo.gl/tH6cnq

Barragán, V., \& Garzón, B. (2017). Radios de las nacionalidades en Ecuador. Un proceso de acción de los derechos a la comunicación. Radios, redes e internet para la transformación social, 8(73). Recuperado de: https:// goo.gl/5VNWbp 
Beltrán, L. R., \& Reyes, J. (1993). Radio popular en Bolivia: la lucha de obreros y campesinos para democratizar la comunicación. Diálogos de la Comunicación, 35, 14-31. Recuperado de: https://goo.gl/oSpvBR

Beltrán, L. (1996). La radio popular y educativa en América Latina. Chasqui. Revista Latinoamericana de Comunicación, 0(53), 08-11. http://dx.doi. org/10.16921/chasqui.v0i53.994

Cerbino, M., \& Belotti, F. (2016). Community Media as Exercise of Communicative Citizenship: Experiences from Argentina and Ecuador. [Medios comunitarios como ejercicio de ciudadanía comunicativa: experiencias desde Argentina y Ecuador]. Comunicar, 47, 49-56. https://doi.org/10.3916/ C47-2016-05

Constituyente, E. A. (2008). Constitución de la República del Ecuador.

De la Noval Bautista, L. A. (2018). La radio comunitaria en función del desarrollo. Revista Estudios del Desarrollo Social: Cuba y América Latina, 6(2), 52-64. Recuperado de: https://goo.gl/2q3rhg

Galán Montesdeoca, J. (2015). Los medios comunitarios, un reto para la comunicación en el Ecuador. Recuperado de: https://goo.gl/gQjrFd

Galarza, V., \& Amador, B. (2018). La radio comunitaria en Imbabura: emisoras, programación y compromiso con la audiencia. Revista científica axioma, (17), 69-77. Recuperado de: https://goo.gl/M9JqLA

Galarza, V., \& Culqui, A. (2017). La radio comunitaria y su legislación en la Comunidad Andina. En Del verbo al bit (pp. 1383-1407). Sociedad Latina de Comunicación Social. DOI: 10.4185/cac116edicion2

García, N., \& Ávila, C. (2016). Nuevos escenarios para la comunicación comunitaria: Oportunidades y amenazas a medios de comunicación y organizaciones de la sociedad civil a partir de la aplicación del nuevo marco regulatorio ecuatoriano. Palabra Clave, 19(1), 271-303.

García, J. G. (2017). Transformaciones y aprendizajes de las radios comunitarias en España: hacia un modelo de radio inclusiva. Anuario electrónico de estudios en Comunicación Social "Disertaciones", 10(1), 30-41.

Gumucio Dagron, A. (2005). Arte de equilibristas: la sostenibilidad de los medios de comunicación comunitarios. Punto Cero, 10(10), 6-19. Recuperado de: https://goo.gl/rTcgVa

Lizondo, N. L. (2018). La comunicación con identidad. Regulaciones y un estudio de caso. Anuario Electrónico de Estudios en Comunicación Social "Disertaciones”, 11(2), 50-65. http://dx.doi.org/10.12804/revistas.urosario. edu.co/disertaciones/a.5745 
López Vigil, J. (1995). ¿Qué hace comunitaria a una radio comunitaria? Chasqui. Revista Latinoamericana de Comunicación, O(52), 51-54. https://doi. org/10.16921/chasqui.v0i52.621

Martínez Ortiz de Zárate, A. (2018). Transformación social a través de la participación en procesos comunicativos comunitarios. En II Congreso Internacional Move. net sobre Movimientos Sociales y TIC (2018), (pp. 268-285). Grupo Interdisciplinario de Estudios en Comunicación, Política y Cambio Social de la Universidad de Sevilla (COMPOLÍTICAS). Recuperado de: https://goo.gl/yz9nGF

Martínez Ruque, H., Yaguana Romero, H., \& Rencoret Quezada, N. (2018). Espacio público y políticas de comunicación: redefinición del término comunitario en Ecuador. Dilemas Contemporáneos: Educación, Política y Valores, 5(2). Recuperado de: https://goo.gl/KBa36

Martínez-Roa, O. G., \& Ortega-Erazo, E. G. (2018). Percepciones y participación en emisoras de radio comunitaria en Nariño-Colombia. Comunicar: Revista Científica de Comunicación y Educación, 26(54), 81-90. https:// doi.org/10.3916/C54-2018-08

Milan, S. (2006). Medios comunitarios y regulación. Una perspectiva de comunicación para el desarrollo. Investigación \& Desarrollo, 14(2). Recuperado de: https://goo.gl/TCKDLV

Mora, C. (2011). Formas de participación en las radios comunitarias habilitadas del Táchira: Estudio de campo. Anuario Electrónico de Estudios en Comunicación Social "Disertaciones", 4(1), Recuperado de: https://goo.gl/S1Kpmb

Navarro Nicoletti, F. (2017). Neoliberalismo y radios comunitarias: los años noventa y la realidad actual. Improntas de la Historia y la Comunicación. https://doi.org/10.24215/24690457e014

Paiz, G. (2016). La Radio Comunitaria ¿Cómo resignificarla hoy? Ciencia e Interculturalidad, 18(1). Recuperado de: https://goo.gl/YECLgw

Peña Sarmiento, M. F. (2012). Voces y Sonidos de la Madre Tierra: Jujunula Makuira, the Radio which Strengthens Social Tissue in La Guajira (Colombia). Anagramas-Rumbos y sentidos de la comunicación, 10(20), 197-212.

Pincheira Muñoz, L. (2013). Radio comunitaria, un espacio educativo no formal en la comunidad. REXE. Revista de Estudios y Experiencias en Educación, 12(24), 183-193. Recuperado de: https://goo.gl/qtsVKT

Ramos Martín, J. (2018). Los medios comunitarios indígenas como construcción de memoria en resistencia en Bolivia. América Latina Hoy, 78, 17-36. https://doi.org/10.14201/alh2018781736 
Ramírez, J. (2014). En Chile ¿Radio comunitaria o ciudadana? Revista LuciérnagaComunicación, 6(12), 118-126. Recuperado de: https://goo.gl/EkQmhR

Romero, M., \& Artigas, W. (2013). Radios comunitarias del municipio Maracaibo: ¿autosostenibles? Revista Científica Teorías, Enfoques y Aplicaciones en las Ciencias Sociales, 6(13), 67-78. Recuperado de: https://goo.gl/ mF29XT

Sanmartín, J., Avelino, A., Reyes, C. y Cruz, M.J. (2017). La estructura mediática de los colectivos sociales en América Latina: los casos de Uruguay, Argentina, Ecuador y Bolivia. Sphera Publica, 2(17), 91-110. Recuperado de: https://goo.gl/s4wCqb

Tamarit, A., \& Cevallos, J., \& Yépez, J. (2014). Radios y comunidades en la región 5 del Ecuador. Existentes y resistencias entre la reterritorialización y las urgencias de una política comunicativa nacional. Razón y Palabra, 18(88). Recuperado de: https://goo.gl/zaySsw

Sánchez, R. (2013). Apuntes para el debate ecuatoriano sobre medios: Distribución de frecuencias, ¿posibilidades para la educomunicación? Alteridad, 8(2), 162-170. https://doi.org/10.17163/alt.v8n2.2013.04

Villamayor, C. (2014). Las radios comunitarias, gestoras de procesos comunicacionales. Mediaciones, 10(12), 88-105. http://dx.doi.org/10.26620/uniminuto.mediaciones.10.12.2014.88-105

UNESCO (1999). La radio. En: Informe Mundial sobre la Comunicación. Los medios frente al desafío de las nuevas tecnologías (pp.139-158. Madrid. Ediciones UNESCO-CINDOC, Fundación Santa María, Recuperado de: https://goo.gl/mH2PU1

Fecha de envío: 2019/05/13; Fecha de aceptación: 2019/07/26;

Fecha de publicación: 2019/09/01 